International Journal of Pure and Applied Mathematics

Volume 98 No. 3 2015, 323-332

ISSN: 1311-8080 (printed version); ISSN: 1314-3395 (on-line version)

url: http://www.ijpam.eu

doi: http://dx.doi.org/10.12732/ijpam.v98i3.4

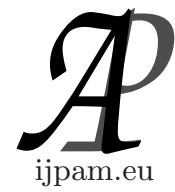

\title{
REMARKS ON GENERALIZED TOPOLOGIES INDUCED BY SUPRA-NEIGHBORHOOD SYSTEMS
}

\author{
Young Key Kim¹, Won Keun Min²§ \\ ${ }^{1}$ Department of Mathematics \\ MyongJi University \\ Youngin, 449-728, KOREA \\ ${ }^{2}$ Department of Mathematics \\ Kangwon National University \\ Chuncheon, 200-701, KOREA
}

\begin{abstract}
We investigate some properties for the generalized topological space induced by a given supra-neighborhood system. And we introduce the

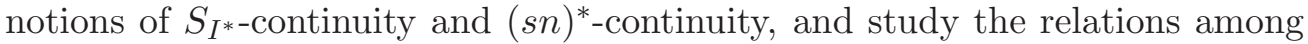

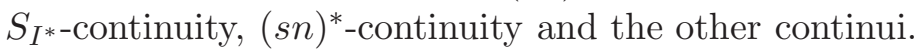

AMS Subject Classification: 54A05, 54C05

Key Words: supra-neighborhood system, supra-neighborhood space, $S_{I^{*-}}$ continuity, $(s n)^{*}$-continuity, $s^{\prime}$-convergence of $m$-family

\section{Introduction}

The notion of generalized neighborhood system (briefly GNS) was introduced by Császár in [1]. He also introduced the notion of $\left(\psi, \psi^{\prime}\right)$-continuity on generalized neighborhood systems $\psi, \psi^{\prime}$. In the same way, we introduced and studied the notion of the weak neighborhood systems in [7]. In [3], we investigated supra-neighborhood systems and supra-neighborhood spaces in order to generalize the notions of GNS, weak neighborhood system [7] and neighborhood structure [2]. In [3], we also introduced the notions of operators $\iota, \gamma, i$ and $c$ in supra-neighborhood spaces, and studied basic properties for such operators.

Received: August 23, 2014

(C) 2015 Academic Publications, Ltd. url: www.acadpubl.eu

$\S$ Correspondence author 
Recently, we investigated two special operators $I^{*}$ and $C l^{*}$ induced by a given supra-neighborhood system in [4]. In this paper, we are going to investigate the collection $\Psi_{I^{*}}$ induced by the operator $I^{*}$. In particular, we introduce

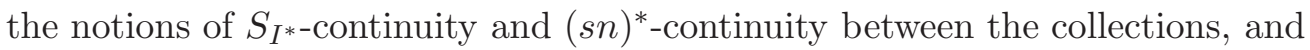

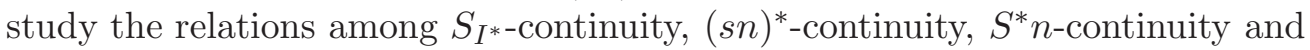
$s^{*}(\psi, \phi)$-continuity.

\section{Preliminaries}

Let $X$ be a nonempty set, $\exp (X)$ the power set of $X$ and $\psi: X \rightarrow \exp (\exp (X))$ satisfy $x \in V$ for $V \in \psi(x)$. Then $V \in \psi(x)$ is called a generalized neighborhood [1] of $x \in X$ and $\psi$ is called a generalized neighborhood system (briefly GNS) on $X$. Let $g$ be a collection of subsets of $X$. Then $g$ is called a generalized topology [1] on $X$ iff $\emptyset \in g$ and $G_{i} \in g$ for $i \in I \neq \emptyset$ implies $G=\cup_{i \in I} G_{i} \in g$. The elements of $g$ are called $g$-open sets and the complements are called $g$-closed sets. For $A \subseteq X$, we denote by $i_{g} A$ the union of all $g$-open sets contained in $A$, i.e. the largest $g$-open set contained in $A$. Any intersection of $g$-closed sets is $g$-closed, and for $A \subseteq X$, we denote by $c_{g} A$ the intersection of all $g$-closed sets containing $A$, i.e. the smallest $g$-closed set containing $A$. Then a function $f: X \rightarrow Y$ is said to be $\left(g_{X}, g_{Y}\right)$-continuous [5] if for each $g_{Y}$-open set $V$ in $Y, f^{-1}(V)$ is $g_{X}$-open in $X$.

Let $\psi: X \rightarrow \exp (\exp (X))$. Then $\psi$ is called a supra-neighborhood system [3] on $X$ if it satisfies the following:

(1) For $x \in X, \psi(x) \neq \emptyset$.

(2) For $V \in \psi(x), x \in V$.

Then the pair $(X, \psi)$ is called a supra-neighborhood space (briefly $S N S$ ) on $X$. Then $V \in \psi(x)$ is called a supra-neighborhood of $x \in X$.

Let $(X, \psi)$ be an SNS on $X$ and $G \subseteq X$. Then $G$ is called an $S^{*}$-open set [3] if for each $x \in G$, there is $V \in \psi(x)$ such that $V \subseteq G$. Let us denote $S^{*}(X)$ the collection of all $S^{*}$-open sets on an SNS $(X, \psi)$. The complements of $S^{*}$-open sets are called $S^{*}$-closed sets. Then we have shown that the collection $S^{*}(X)$ of all $S^{*}$-open subsets of $X$ is a supratopology [5] on $X$, that is, $X \in S^{*}(X)$ and $S^{*}(X)$ is closed under arbitrary union.

We recall the notions of interior operators $i, \iota$ and closure operators $c, \gamma$ introduced in [3], respectively: Let $(X, \psi)$ be an SNS on $X$ and $A \subseteq X$. Then

(1) $i(A)=\cup\left\{G \subseteq: G \subseteq A\right.$ and $G \in S^{*}(X)$;

(2) $c(A)=\cap\left\{F \subseteq: A \subseteq F\right.$ and $X-F \in S^{*}(X)$;

(3) $\iota(A)=\{x \in A$ : there exists $V \in \psi(x)$ such that $V \subseteq A\}$; 
(4) $\gamma(A)=\{x \in X: V \cap A \neq \emptyset$ for all $V \in \psi(x)\}$.

Theorem $2.1([3])$. Let $(X, \psi)$ be an SNS on $X$ and $A \subseteq X$. Then

(1) $i(A) \subseteq \iota(A) \subseteq A$;

(2) $A \subseteq \gamma(A) \subseteq c(A)$;

(3) $A$ is $S^{*}$-open iff $i(A)=A$;

(4) $A$ is $S^{*}$-closed iff $c(A)=A$.

Remark 2.2. Let $(X, \psi)$ and $(Y, \phi)$ be two SNS's. Then $f: X \rightarrow Y$ is said to be

(1) $s^{*}(\psi, \phi)$-continuous [3] if for each $x \in X$ and $V \in \phi(f(x))$, there is $U \in \psi(x)$ such that $f(U) \subseteq V$;

(2) sn-continuous [3] if for every $A \in S^{*}(Y), f^{-1}(A)$ is in $S^{*}(X)$;

(3) $S^{*} n$-continuous [4] if for every $A \in \phi(f(x)), f^{-1}(A)$ is in $\psi(x)$.

$$
S^{*} n \text {-continuous } \Rightarrow s^{*}(\psi, \phi) \text {-continuous } \Rightarrow s n \text {-continuous }
$$

\section{Main Results}

We recall two special operators $I^{*}$ and $C l^{*}$ introduced in [4]: Let $(X, \psi)$ be an SNS and $A \subseteq X$.

$$
I^{*}(A)=\{x \in A: A \in \psi(x)\} ; \quad C l^{*}(A)=\{x \in X: X-A \notin \psi(x)\} .
$$

In [4], we showed that the operators satisfy $I^{*}(A) \subseteq A \subseteq C l^{*}(A)$ but the following statements are not always true:

(1) If $A \subseteq B$, then $I^{*}(A) \subseteq I^{*}(B) ; C l^{*}(A) \subseteq C l^{*}(B)$.

(2) $I^{*}(A) \cap I^{*}(B)=I^{*}(A \cap B) ; \quad C l^{*}(A) \cup C l^{*}(B)=C l^{*}(A \cup B)$.

(3) $I^{*}\left(I^{*}(A)\right)=I^{*}(A) ; \quad C l^{*}\left(C l^{*}(A)\right)=C l^{*}(A)$.

From now on, we study the collection induced by the operator $I^{*}$ :

Definition 3.1. Let $(X, \psi)$ be an SNS on $X$ and $\Psi_{I^{*}}(X)=\{\cup \sigma: \sigma \subseteq \mathbf{B}\}$ where $\mathbf{B}=\left\{A \subseteq X: I^{*}(A)=A\right\}$. For $G \subseteq X, G$ is called an $S_{I^{*-o p e n ~ s e t ~}}$ if $G \in \Psi_{I^{*}}(X)$.

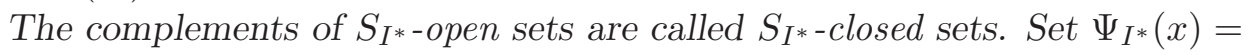
$\left\{G \in \Psi_{I^{*}}(X): x \in G\right\}$.

Remark 3.2. Let $(X, \psi)$ be an SNS on $X$. Then clearly $\Psi_{I^{*}}(X)$ is a generalized topology on $X$ but it need not be a supra-topology (See Example 3.16 in $[4])$. 
Definition 3.3. Let $(X, \psi)$ be an SNS on $X$ and $A \subseteq X$. The $S_{I^{*-}}$ interior of $A$, denoted by $i^{*}(A)$, is the union of all $G \subseteq A, G \in \Psi_{I^{*}}(X)$, and

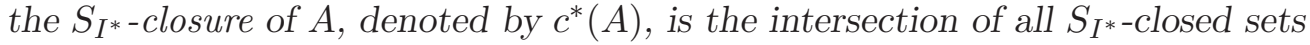
containing $A$.

Theorem 3.4. Let $(X, \psi)$ be an SNS on $X$ and $A \subseteq X$. Then the following hold.

(1) $i^{*}(A) \subseteq i(A) \subseteq A$;

(2) $A \subseteq c^{*}(A) \subseteq c(A)$;

(3) $A$ is $S_{I^{*}-o p e n}$ if and only if $i^{*}(A)=A$;

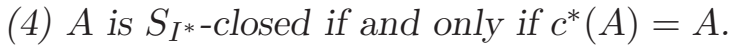

Proof. (1) We show that $i^{*}(A) \subseteq i(A)$. Let $x \in i^{*}(A)$. Then there exists an $S_{I^{*}-\text { open set }} G$ such that $x \in G \subseteq A$. From the definition of $S_{I^{*-o p e n n e s s,}}$ there is a family $\sigma=\left\{U \subseteq X: I^{*}(U)=U\right\}$ such that $G=\cup \sigma$. Let $U \in \sigma$. For each $z \in U$, from $z \in U=I^{*}(U)$, it follows $U \in \psi(z)$ and $z \in i(U)$, and so $U$ is $S^{*}$-open. This implies $G$ is $S^{*}$-open and $x \in i(A)$. So from Theorem 2.1, the inclusion relations are completed.

(2) It is similar to (1).

(3) and (4) are obvious.

Example 3.5. Let $X=\{a, b, c, d\}$ and $\psi: X \rightarrow \exp (\exp (X))$ a supraneighborhood system defined as the following: $\psi(a)=\{\{a, c\}\}, \psi(b)=\{\{b\}\}$, $\psi(c)=\psi(d)=\{X\}$. Then for $A=\{a, b, c\} \subseteq X, i(A)=\{a, b\}$ and $i^{*}(A)=\emptyset$, and so $i^{*}(A) \neq i(A)$.

Theorem 3.6. Let $(X, \psi)$ be an SNS on $X$. Then every $S_{I^{*-o p e n}}$ set is $S^{*}$-open.

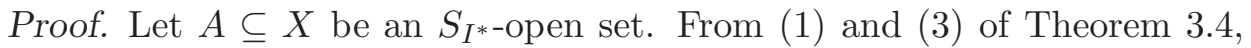
$i(A)=A$ is obtained. It implies $A$ is $S^{*}$-open.

We recall the notion of $m$-family [6]: A collection $\mathbf{H}$ of subsets of $X$ is called an $m$-family on $X$ if $\cap \mathbf{H}=\cap\{G: G \in \mathbf{H}\} \neq \emptyset$.

Definition 3.7. Let $(X, \psi)$ be an SNS and let $\mathbf{H}$ be an $m$-family on $X$. Then we say that an $m$-family $\mathbf{H} s^{*}$-converges to $x \in X$ if $\Psi_{I^{*}}(x) \subseteq \mathbf{H}$.

Theorem 3.8. Let $(X, \psi)$ be an SNS and $A \subseteq X$. Then the following hold.

(1) $i^{*}(A)=\left\{x \in A: A \in \mathbf{H}\right.$, for every $m$-family $\mathbf{H} s^{*}$-converging to $\left.x\right\}$.

(2) $c^{*}(A)=\left\{x \in X\right.$ : there exists an $m$-family $\mathbf{H}$ such that $\mathbf{H} s^{*}$-converges to $x$ and $A \in \mathbf{H}\}$. 
Proof. (1) Let $x \in i^{*}(A)$ and an $m$-family $\mathbf{H} s^{*}$-converge to $x$. Then it is obvious that $A \in \Psi_{I^{*}}(x) \subseteq \mathbf{H}$.

Suppose that for every $m$-family $\mathbf{H} s^{*}$-converging to $x, A \in \mathbf{H}$. Since $\Psi_{I^{*}}(x)$ $s^{*}$-converges to $x$, by hypothesis, $A \in \Psi_{I^{*}}(x)$, so that $x \in i^{*}(A)$.

(2) Let $x \in C l^{*}(A)$; then for all $V \in \Psi_{I^{*}}(x), V \cap A \neq \emptyset$. Set $\mathbf{H}=$ $\Psi_{I^{*}}(x) \cup\{A\}$; then $\mathbf{H}$ is an $m$-family $s^{*}$-converging to $x$ such that $A \in \mathbf{H}$.

For the converse, let $\mathbf{H}$ be an $m$-family $s^{*}$-converging to $x$ and $A \in \mathbf{H}$; then since $\Psi_{I^{*}}(x)$ is contained in $\mathbf{H}$, by definition of $m$-family, $V \cap A \neq \emptyset$ for all $V \in \Psi_{I^{*}}(x)$. Hence $x \in c^{*}(A)$.

Theorem 3.9. Let $(X, \psi)$ be a generalized topological space and $A \subseteq X$. Then the following hold.

(1) $i_{g}(A)=\left\{x \in A: A \in \mathbf{H}\right.$, for every $m$-family $\mathbf{H} s^{*}$-converging to $\left.x\right\}$.

(2) $c_{g}(A)=\left\{x \in X\right.$ : there exists an $m$-family $\mathbf{H}$ such that $\mathbf{H} s^{*}$-converges to $x$ and $A \in \mathbf{H}\}$.

Proof. First, for $A \subseteq X$, we know that $i^{*}(A)\left(c^{*}(A)\right)$ is generalized open (generalized close), since the family $\Psi_{I^{*}}=\{\cup \sigma: \sigma \subseteq \mathbf{B}\}$ is a generalized topology. So from Theorem 3.8, the above statements (1) and (2) are directly obtained.

Definition 3.10. Let $f: X \rightarrow Y$ be a function on two SNS's $(X, \psi)$ and

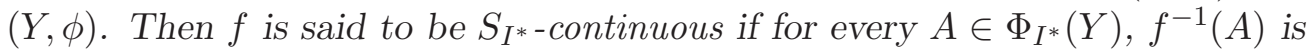
in $\Psi_{I^{*}}(X)$.

Theorem 3.11. Let $f: X \rightarrow Y$ be a function on two SNS's $(X, \psi)$ and $(Y, \phi)$. Then the following things are equivalent:

(1) $f$ is $S_{I^{*}-\text { continuous. }}$

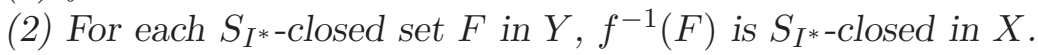

(3) $f\left(c^{*}(A)\right) \subseteq c^{*}(f(A))$ for all $A \subseteq X$.

(4) $c^{*}\left(f^{-1}(B)\right) \subseteq f^{-1}\left(c^{*}(B)\right)$ for all $B \subseteq Y$.

(5) $f^{-1}\left(i^{*}(B)\right) \subseteq i^{*}\left(f^{-1}(B)\right)$ for all $B \subseteq Y$.

(6) $f:\left(X, \Psi_{I^{*}}(X)\right) \rightarrow\left(Y, \Phi_{I^{*}}(Y)\right)$ is $\left(\Psi_{I^{*}}(X), \Phi_{I^{*}}(Y)\right)$-continuous.

Proof. Obvious.

Corollary 3.12. Let $f: X \rightarrow Y$ be a function on generalized topological spaces $\left(X, g_{X}\right)$ and $\left(Y, g_{Y}\right)$. Then the following things are equivalent:

(1) $f$ is $\left(g_{X}, g_{Y}\right)$-continuous.

(2) For each $g$-closed set $F$ in $Y, f^{-1}(F)$ is $g$-closed in $X$. 
(3) $f\left(c_{g}(A)\right) \subseteq c_{g}(f(A))$ for all $A \subseteq X$.

(4) $c_{g}\left(f^{-1}(B)\right) \subseteq f^{-1}\left(c_{g}(B)\right)$ for all $B \subseteq Y$.

(5) $f^{-1}\left(i_{g}(B)\right) \subseteq i_{g}\left(f^{-1}(B)\right)$ for all $B \subseteq Y$.

Proof. Since $\Psi_{I^{*}}(X)$ and $\Phi_{I^{*}}(Y)$ are generalized topologies on $X$ and $Y$, respectively, the corollary is obtained from Theorem 3.11.

Theorem 3.13. Let $f: X \rightarrow Y$ be a function on two $\operatorname{SNS}^{\prime} \mathrm{S}(X, \psi)$ and $(Y, \phi)$. If $f$ is $S^{*} n$-continuous, then it is also $S_{I^{*}-\text { continuous. }}$

Proof. Let $A \in \Phi_{I^{*}}(Y)$. Then $A=\cup A_{\alpha}$ where $I^{*}\left(A_{\alpha}\right)=A_{\alpha}$. First, we show that for each $\alpha, I^{*}\left(f^{-1}\left(A_{\alpha}\right)\right)=f^{-1}\left(A_{\alpha}\right)$. For the proof, let $x \in f^{-1}\left(A_{\alpha}\right)$; then $f(x) \in A_{\alpha}=I^{*}\left(A_{\alpha}\right)$, and $A_{\alpha} \in \phi(f(x))$. From $S^{*} n$-continuity of $f$, it is obtained $f^{-1}\left(A_{\alpha}\right) \in \psi(x)$. It implies $x \in I^{*}\left(f^{-1}\left(A_{\alpha}\right)\right)$ and $I^{*}\left(f^{-1}\left(A_{\alpha}\right)\right)=$ $f^{-1}\left(A_{\alpha}\right)$. From this fact, we have $f^{-1}(A)=\cup f^{-1}\left(A_{\alpha}\right)$ such that $I^{*}\left(f^{-1}\left(A_{\alpha}\right)\right)=$

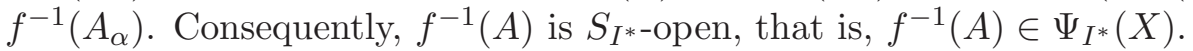

In the next example, we can show that the converse is not always true:

Example 3.14. Let $X=\{a, b, c, d\}$ and let $\psi: X \rightarrow \exp (\exp (X))$ be a supra-neighborhood system defined as Example 3.5. Consider a function $f:(X, \psi) \rightarrow(X, \psi)$ as follows $f(a)=a, f(b)=b, f(c)=d, f(d)=c$.

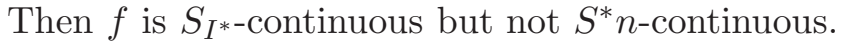

Moreover, we can show that there is no any relation between $S_{I^{*} \text {-continuity }}$ and $s^{*}(\psi, \phi)$-continuity as in the next example:

Example 3.15. Let $X=\{a, b, c, d\}$ and let $\psi: X \rightarrow \exp (\exp (X))$ be a supra-neighborhood system defined as Example 3.5.

(1) Let us define the function $f:(X, \psi) \rightarrow(X, \psi)$ as follows

$$
f(a)=a, f(b)=b, f(c)=d, f(d)=c .
$$

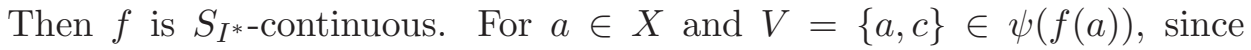
$\{a, c\} \in \psi(a)$ and $f(\{a, c\})=\{a, d\}$, there is no $U \in \psi(a)$ such that $f(U) \subseteq V$, so $f$ is not $s^{*}(\psi, \psi)$-continuous.

(2) Let us define the function $f:(X, \psi) \rightarrow(X, \psi)$ as follows

$$
f(a)=f(c)=b, f(b)=c, f(d)=d .
$$

Then $f$ is $s^{*}(\psi, \psi)$-continuous. For the $S_{I^{*-o p e n}}$ set $G=\{b\}, f^{-1}(G)=$ $\{a, c\}$ and it is not $S_{I^{*-o p e n}}$. So $f$ is not $S_{I^{*} \text {-continuous. }}$

In the next examples, we show that there is no any relationship between $S_{I^{*}-\text { continuity and } s n \text {-continuity: }}$ 
Example 3.16. Let $X=\{a, b, c, d\}$. Consider a supra-neighborhood system $\psi: X \rightarrow \exp (\exp (X))$ defined as the following: $\psi(a)=\{\{a, b\}\}, \psi(b)=$ $\psi(c)=\{\{b, c\}\}$, and $\psi(d)=\emptyset$. Note that $\Psi_{I^{*}}(X)=\{\emptyset,\{b, c\}\}$ and $S^{*}(X)=$ $\{\emptyset,\{b, c\},\{a, b, c\}\}$.

(1) Let us define the function $f:(X, \psi) \rightarrow(X, \psi)$ as the following:

$$
f(a)=a, f(b)=c, f(c)=b, f(d)=a .
$$

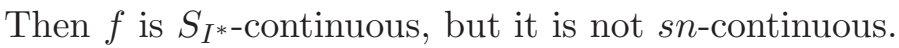

(2) Let us define the function $g:(X, \psi) \rightarrow(X, \psi)$ as the following:

$$
g(a)=c, g(b)=c, g(c)=b, g(d)=d .
$$

Then $g$ is $s n$-continuous, but it is not $S_{I^{*} \text {-continuous. }}$

Finally, from the above examples and Remark 2.3, the following diagram is obtained:

$$
\begin{aligned}
& S^{*} n \text {-continuous } \\
& \Downarrow \\
& S_{I^{*} \text {-continuous }}
\end{aligned} \Rightarrow s^{*}(\psi, \phi) \text {-continuous } \Rightarrow s n \text {-continuous }
$$

Theorem 3.17. Let $f: X \rightarrow Y$ be a bijective function between SNS's

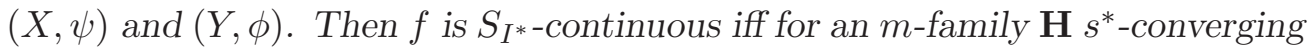
to $x \in X, f(\mathbf{H}) s^{*}$-converges to $f(x)$.

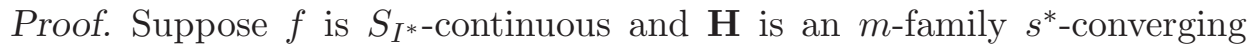
to $x \in X$. It is obvious $f(\mathbf{H})=\{f(F): F \in \mathbf{H}\}$ is an $m$-family on $Y$. By hypothesis and surjectivity, we get $\Phi_{I^{*}}(f(x)) \subseteq f\left(\Psi_{I^{*}}(x)\right) \subseteq f(\mathbf{H})$, so that $f(\mathbf{H}) s^{*}$-converges to $f(x)$.

For the converse, let $G \in \Phi_{I^{*}}(f(x))$ for $G \subseteq Y$. Since $\Psi_{I^{*}}(x) s^{*}$-converges to $x$, by hypothesis, we get $\Phi_{I^{*}}(f(x)) \subseteq f\left(\Psi_{I^{*}}(x)\right)$ for $x \in X$. Since $f$ is injective, $f^{-1}(G) \in \Psi_{I^{*}}(x)$. Hence $f$ is $S_{I^{*}-\text { continuous. }}$

From Theorem 3.17, we have the following:

Corollary 3.18. Let $f: X \rightarrow Y$ be a bijective function between generalized topological spaces $\left(X, g_{X}\right),\left(Y, g_{Y}\right)$. Then $f$ is $\left(g_{X}, g_{Y}\right)$-continuous iff for an $m$-family $\mathbf{H} s^{*}$-converging to $x \in X, f(\mathbf{H}) s^{*}$-converges to $f(x)$.

Definition 3.19. Let $f: X \rightarrow Y$ be a function on two SNS's $(X, \psi)$ and $(Y, \phi)$. Then $f$ is said to be $(s n)^{*}$-continuous if for every $S_{I^{*}-o p e n}$ set $A$ in $Y$, $f^{-1}(A)$ is $S^{*}$-open in $X$. 
Theorem $3.20([3])$. Let $f: X \rightarrow Y$ be a function on two SNS's $(X, \psi)$ and $(Y, \phi)$. Then the following things are equivalent:

(1) $f$ is sn-continuous.

(2) For each $S^{*}$-closed set $F$ in $Y, f^{-1}(F)$ is $S^{*}$-closed in $X$.

(3) $f(c(A)) \subseteq c(f(A))$ for all $A \subseteq X$.

(4) $c\left(f^{-1}(B)\right) \subseteq f^{-1}(c(B))$ for all $B \subseteq Y$.

(5) $f^{-1}(i(B)) \subseteq i\left(f^{-1}(B)\right)$ for all $B \subseteq Y$.

Theorem 3.21. Let $f: X \rightarrow Y$ be a function on two SNS's $(X, \psi)$ and $(Y, \phi)$. Then the following things are equivalent:

(1) $f$ is $(s n)^{*}$-continuous.

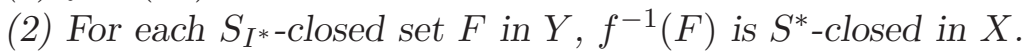

(3) $f(c(A)) \subseteq c^{*}(f(A))$ for all $A \subseteq X$.

(4) $c\left(f^{-1}(B)\right) \subseteq f^{-1}\left(c^{*}(B)\right)$ for all $B \subseteq Y$.

(5) $f^{-1}\left(i^{*}(B)\right) \subseteq i\left(f^{-1}(B)\right)$ for all $B \subseteq Y$.

Proof. It follows from Theorem 3.11 and Theorem 3.20.

Theorem 3.22. Let $f: X \rightarrow Y$ be a function on two $\operatorname{SNS}^{\prime}$ S $(X, \psi)$ and $(Y, \phi)$. Then:

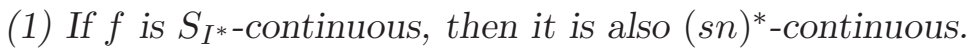

(2) If $f$ is sn-continuous, then it is also $(s n)^{*}$-continuous.

Proof. It is obvious from Theorem 3.6 and definitions of such continuous functions.

In Theorem 3.22, the converses may not be true as the next example:

Example 3.23. Let $X=\{a, b, c, d\}$. Consider a supra-neighborhood system $\psi: X \rightarrow \exp (\exp (X))$ defined as the following: $\psi(a)=\{\{a, b\}\}, \psi(b)=$ $\psi(c)=\{\{b, c\}\}$, and $\psi(d)=\emptyset$. Note that $\Psi_{I^{*}}(X)=\{\emptyset,\{b, c\}\}$ and $S^{*}(X)=$ $\{\emptyset,\{b, c\},\{a, b, c\}\}$.

(1) Let us define the function $f:(X, \psi) \rightarrow(X, \psi)$ as follows

$$
f(a)=b, f(b)=b, f(c)=b, f(d)=a .
$$

Then $f$ is $(s n)^{*}$-continuous, but it is not $s n$-continuous.

(2) Let us define the function $g:(X, \psi) \rightarrow(X, \psi)$ as follows

$$
g(a)=c, g(b)=c, g(c)=b, g(d)=d .
$$

Then $g$ is $(s n)^{*}$-continuous, but it is not $S_{I^{*} \text {-continuous. }}$

In summary, we have the following implications: 


$$
\begin{aligned}
& S^{*} n \text {-continuous } \Rightarrow s^{*}(\psi, \phi) \text {-continuous } \Rightarrow s n \text {-continuous } \\
& \Downarrow \\
& S_{I^{*} \text {-continuous }} \quad \Rightarrow \quad(s n)^{*} \text {-continuous }
\end{aligned}
$$

\section{Conclusion}

We investigated some properties for the generalized topology induced by a given supra-neighborhood system on $X$. And we introduced the notions of $S_{I^{*-}}$ continuity and $(s n)^{*}$-continuity, and studied the relations among $S_{I^{*} \text {-continuity, }}$ $(s n)^{*}$-continuity, $S^{*} n$-continuity and $s^{*}(\psi, \phi)$-continuity. In the next research, we are going to study a soft generalized topological unverse induced by soft supra-neighborhood systems on a universe set, and investigate soft $S_{I^{*}-\text { continuity }}$ and soft $(s n)^{*}$-continuity.

\section{Acknowledgments}

This research was supported by Basic Science Research Program through the National Research Foundation of Korea(NRF) funded by the Ministry of Education, Science and Technology(Grant No. NRF-2012R1A1A4A01004765)

\section{References}

[1] Á. Császár, Generalized Topology, Generalized Continuity, Acta Math. Hungar., 96 (2002), 351-357.

[2] D. C. Kent and W. K. Min, Neighborhood Spaces, International Journal of Mathematics and Mathematical Sciences, 32(7) (2002), 387-399.

[3] Y. K. Kim and W. K. Min, On Supra-neighborhood Systems and Spaces, Far East Journal of Mathematical Sciences, 80(2) (2013), 261-272.

[4] Y. K. Kim and W. K. Min, Some Results On Supra-Neighborhood Spaces, Submitted.

[5] A. S. Mashhourr, A. A. Allam, F. S. Mahmoud and F. H. Khadr, On Supratopological Spaces, Indian J. Pure Appl. Math., 14(4) (1983), 502510. 
[6] W. K. Min, Some Results on Generalized Topological Spaces and Generalized Systems, Acta Math. Hungar., 108 (1-2) (2005), 171-181.

[7] W. K. Min, On Weak Neighborhood Systems and Spaces, Acta Math. Hungar., 121 (3) (2008), 283-292. 